\title{
Desigualdad y Reforma estructural tributaria en Colombia*. Hacia una economía política de inclusión social**
}

Luis Jorge Garay S.*** y Jorge Enrique Espitia Z. ${ }^{* * * * *}$

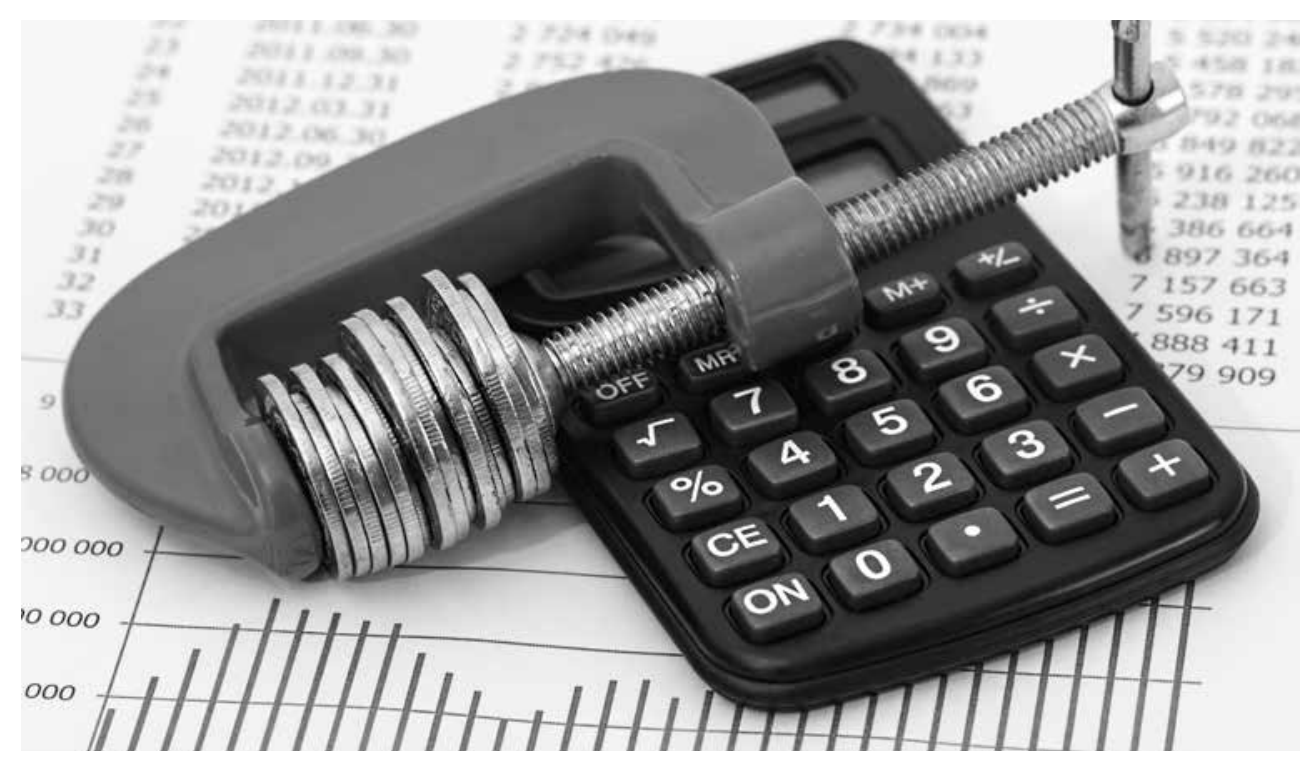

\section{Introducción: a manera de síntesis conclusiva}

Como continuación del programa de investigación sobre las desigualdades en Colombia, iniciado como una primera etapa con el libro de los autores La dinámica de las desigualdades en Colombia (Ediciones Desde Abajo, noviembre 2019), el propósito de las Notas de investigación incluidas en este segundo volumen es el de profundizar en el análisis de diversas temáticas sobre la desigualdad en los ámbitos socioeconómico y tributario en Colombia.

El propósito básico de las primeras siete Notas de investigación es profundizar en el análisis de la desigualdad y la dispersión en la distribución y composición de

Citar este artículo como: Garay S., L. J. y Espitia Z., J. E. (2020). Desigualdad y Reforma estructural tributaria en Colombia. Hacia una economía política de inclusión social. Revista Papeles, 11(22), 154-160.

Fecha de recibido: octubre 10 de 2019 - Fecha de aceptación: diciembre 15 de 2019

* Los autores han cedido a la Revista Papeles este fragmento de su más reciente libro.

** Garay, L. J. y Espitia J. E. (2020). Desigualdad y Reforma estructural tributaria en Colombia. Hacia una economía política de inclusión social. Bogotá: Ediciones Desde Abajo.

** Ingeniero industrial de la Universidad de los Andes, MA en economía de la misma universidad y PhD del MIT. Correo electrónico: ljgara@yahoo.com.mx

*\%: Ingeniero eléctrico de la ECI, economista de la UNAL. Correo electrónico: jeespitia@gmail.com 
la riqueza y de los ingresos tanto de las personas jurídicas como de las personas naturales del país en 2017 con base en la información oficial de la Dian, no solo a nivel agregado sino, especialmente, a nivel de patrimonio e ingreso, partiendo de términos como deciles, y desagregando sucesivamente el $10 \%$ de las personas jurídicas y naturales ricas - pertenecientes al decil 10- en subdeciles 1 a 10, para discriminar, a su vez, el $1 \%$ de las personas naturales y jurídicas superricas - pertenecientes al subdecil 10 del decil 10 - en subdeciles 1 a 10, hasta identificar el $0.1 \%$ de las personas jurídicas y naturales naturales super superricas -pertenecientes al subdecil 10 del subdecil 10 del decil 10.

Con ello se busca trascender cualquier medida sintética de desigualdad como el índice de Gini, para avanzar en la comprensión de las características básicas de la distribución de riqueza e ingresos como requisito básico para la comprensión de los rasgos distintivos de las desigualdades diferenciales que puedan reproducirse a todo lo largo de la distribución de personas naturales y jurídicas, según sus niveles de patrimonio e Ingreso. Para ello se parte del análisis por deciles que cobija cada uno del $10 \%$ de las personas clasificadas según su nivel relativo de patrimonio e ingresos, seguido por el de los subdeciles del $10 \%$ de las personas ricas - pertenecientes al decil $10-y$ por el de los subdeciles del $1 \%$ de las personas superricas - pertenecientes al subdecil 10 del decil 10-, hasta llegar al $0.1 \%$ de las personas super superricas del país - pertenecientes al subdecil 10 del subdecil 10 del decil 10.

Dos de las conclusiones a las que se llegan son las siguientes: se observan tanto diferencias importantes en la composición del patrimonio y de los ingresos de las personas a lo largo de la distribución, así como niveles altos de desigualdad y de dispersión en ingresos y riqueza, incluso en el caso de las personas superricas y aun en el caso de las super superricas.

Las Notas de investigación 8, 9 y 10 tienen por finalidad cuantificar $y$ analizar los diferentes componentes del denominado gasto tributario por concepto del impuesto a la renta en el caso de personas jurídicas y naturales en el año 2017, de acuerdo con la base de información de la Dian, discriminado según el sector económico y nivel de ingreso bruto (en el caso de personas jurídicas) y según el nivel de ingreso bruto (en el de las personas naturales). Ello se hace para poder comprehender la importancia y magnitud del recaudo por concepto del impuesto a la renta y complementarios que deja de percibir el gobierno nacional debido a las exenciones, descuentos y otros tratos preferenciales y discriminatorios que establece el estatuto tributario, así como para poder identificar los sectores y tipos de personas, jurídicas y naturales según su nivel de ingreso, que resultan beneficiadas con esos tratos preferenciales (gran número de ellos sin una debida justificación social ni económica).

Aquí reside una de las raíces de la dinámica de reproducción de desigualdades, dada la peculiaridad de que estos tratos discriminatorios muestran un claro sesgo prorrico, especialmente pro superrico y pro super superrico.

Las Notas de investigación 11 y 12 buscan comparar las estructuras de tarifas efectivas del impuesto a la renta para personas jurídicas según el sector y el nivel de ingreso bruto en 2017 y 2018, estimadas de acuerdo con la información de la Dian y publicadas en el primer volumen de este libro (mencionado al comienzo de esta introducción), con aquellas inferidas de los datos de las empresas que reportaron a Supersociedades en 2018, con el propósito de evaluar la relativa consistencia entre las dos fuentes de información y de constatar una estructura suficientemente representativa para realizar análisis tributarios. En el caso de las personas jurídicas del sector financiero, las que no reportaron a Supersociedades sino a Superfinanciera, se realizó la misma comparación a partir de la base de datos de esta última entidad. 
Si bien hay diferencias entre las bases de datos, en términos de su cobertura a empresas diferenciales entre sectores y en los criterios de definición de muchos de los rubros por el carácter más de tipo económico de las declaraciones de renta ante la Dian (y eminentemente financiero de los reportes ante Supersociedades y Superfinanciera), ha de resaltarse una relativa correspondencia tanto en los niveles promedio y de dispersión de las tarifas efectivas para el conjunto de personas jurídicas y de empresas, como en el ordenamiento de tarifas efectivas entre varios de los sectores en referencia.

Una característica esencial, y que es ampliamente corroborada, es el claro sesgo prorrico, especialmente pro superrico y pro super superrico, de la tarifa efectiva del impuesto de renta para las personas jurídicas.

La Nota de investigación 13 presenta un análisis de la progresividad y regresividad del impuesto a la renta de las personas naturales y jurídicas con base en diversos indicadores planteados en la literatura especializada, como complemento al análisis desarrollado en Garay y Espitia (2019, cap. 3).

Las Notas de investigación 14, 15 y 16 pretenden identificar algunos de los factores determinantes de la tasa de ganancia empresarial; todo ello con base en la estimación de algunos modelos econométricos básicos por mínimos cuadrados, en los que se incluyen, como variables independientes, la tarifa efectiva del impuesto a la renta para personas jurídicas, la participación de los costos de nómina en los costos totales y el poder de

\section{El hallazgo más significativo es que} en el caso del sector manufacturero el poder de mercado está positivamente relacionado con la tasa de ganancia, en tanto que la tarifa efectiva lo está negativamente. mercado de las principales empresas de cada sector en términos de ingresos operacionales -como proxi del nivel de concentración propuesto por J. S. Bain en el campo de la organización industrial.

El hallazgo más significativo es que en el caso del sector manufacturero el poder de mercado está positivamente relacionado con la tasa de ganancia, en tanto que la tarifa efectiva lo está negativamente, como es de esperar -al menos en principio-, según la tesis de Bain y la hipótesis de costos en condiciones de competencia. Con el ingrediente adicional de que una alta proporción -más del $55 \%$ - del comportamiento de la tasa de ganancia podría ser explicada estadísticamente por estos factores determinantes.

Ahora bien, en otros sectores como el minero y financiero no se cumplen ni la tesis de Bain ni la hipótesis de costos porque, entre otras razones, se trata de sectores con niveles muy elevados de concentración de mercado en escaso número de empresas y personas jurídicas. Esto se debe a que, en el primer caso, la explotación de una mina por sus condiciones intrínsecas de especificidad y de ausencia de estandarización no se da en sí misma en el marco de un verdadero mercado de competencia; y también a que, en el segundo, su especialización es la provisión de servicios financieros y no de bienes y mercancías tangibles resultantes de procesos productivos consistentes en la transformación de insumos y materias primas, como de los que trata la teoría tradicional de la competencia.

No obstante, variables como poder de mercado y tarifa efectiva del impuesto a la renta podrían explicar incluso más del $69 \%$ del comportamiento de la tasa de ganancia empresarial.

De otra parte, es de relievar el hecho que resulta posible inferir diferencias sustanciales en los determinantes de las tasas de ganancia de las personas jurídicas más ricas del país, especialmente las superricas y las super superricas; al punto de que para estos últimos 
grupos de personas jurídicas la tarifa efectiva del impuesto a la renta no afecta significativamente la tasa de ganancia y, además, que la intensidad de costos salariales llega a incidir positivamente en la tasa de ganancias, como si los ingresos salariales vinieran a ser parte, al menos indirectamente, de las ganancias de dichas empresas, debido a que, por ejemplo, la remuneración de los superejecutivos podría constituir una porción implícita de las ganancias empresariales. Esta última temática fue introducida por Piketty en su libro Capital en el siglo XXI (2014 [Garay y Espitia, 2019, cap. 1]).

Ante la existencia de otros impuestos adicionales al impuesto a la renta y complementarios y de otras cargas, como los aportes tanto a la seguridad cocial como a las entidades (cajas de compensación, ICBF, etc.), que recaen sobre las personas jurídicas, en las Notas de investigación 17 y 18 se procede a evaluar las tarifas efectivas globales para las personas jurídicas, con base en la información de la Dian del 2018, y también a analizar su estructura a nivel económico y según el nivel de ingreso.

Se entiende por tarifa efectiva global como la tarifa efectiva para la suma del total de Impuestos - a la renta y otros impuestos-, y de los aportes tanto a la seguridad social como a las entidades, medida en términos de ingresos brutos o de ingresos brutos netos de costos de producción.

Sobresalen dos características básicas de la estructura de tarifas efectivas globales según los ingresos brutos netos de costos de producción de las personas jurídicas en 2018. A saber: (i) una elevada heterogeneidad entre sectores, al punto de variar entre una tarifa efectiva del $5.28 \%$ para el sector de actividades financieras $^{1}$, a unas cercanas al $20 \%$ para actividades

1 Esta tarifa efectiva global del sector financiero, con base en los datos de la Dian, se aproxima suficientemente a la estimada de acuerdo con la información de Superfinanciera para 2018.

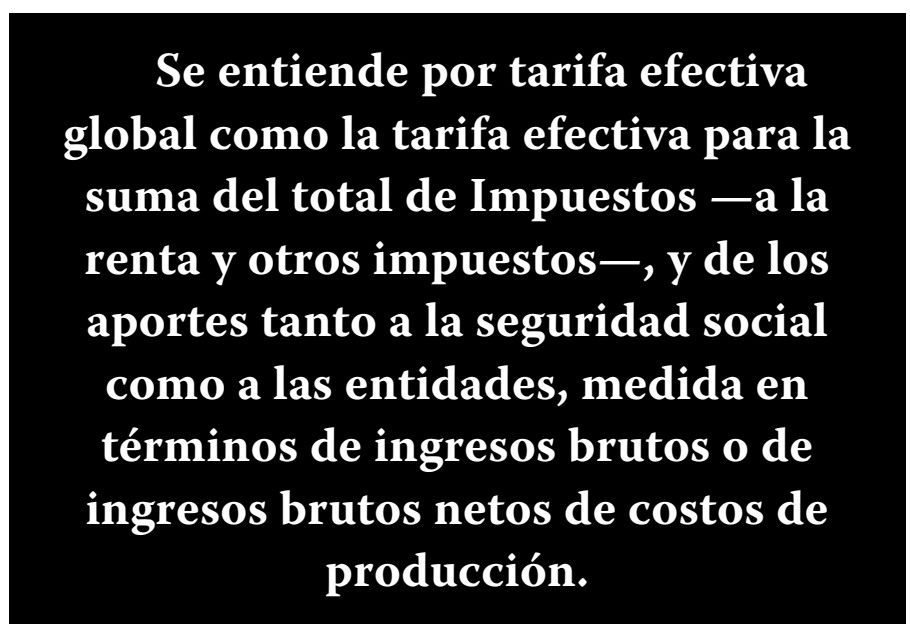

como las de transporte, comunicación y agropecuarias; y hasta una del $32.32 \%$ para el de minería - jalonada por el caso excepcional de Ecopetrol en la actividad de extracción de petróleo-, con una tarifa efectiva promedio para el conjunto de personas jurídicas de 12.11 $\%^{2}$; y (ii) una alta dispersión de las tarifas efectivas globales a nivel sectorial - esto es, altos coeficientes de variación medidos por la relación entre desviación estándar y media de las tarifas efectivas a este nivel-, que resultan de la diversidad de beneficios fiscales otorgados por el Estatuto Tributario entre actividades de un mismo sector.

Además, se constata una vez más el marcado sesgo prorrico, especialmente pro superrico y pro super superrico, de la tarifa efectiva global de las personas jurídicas en el país, algo que contraviene los principios constitucionales de equidad horizontal y progresividad vertical que deberían regir la estructura tributaria en el país.

En la Nota de investigación 19 se retoma el análisis de las Notas de investigación 13, 14 y 15 , especialmente el de la última (sobre determinantes potenciales de la tasa de ganancia de las personas jurídicas), considerando entre ellos la tarifa efectiva global en lugar de la tarifa

$2 \mathrm{Y}$ con una tarifa efectiva global promedia según los ingresos brutos del $4.62 \%$ para el conjunto de personas jurídicas. 
efectiva del impuesto a la renta y al poder de mercado. Los resultados de la estimación de los modelos econométricos básicos a nivel sectorial son muy similares, específicamente en los casos de sectores como el manufacturero y el minero, por lo que podría inferirse que los impactos de la tarifa efectiva resultarían relativamente asimilables entre la global y la del impuesto a la renta.

A su vez, la tarifa efectiva global está relacionada, en sentido opuesto al esperado - al menos inicialmente-, con la tasa de ganancia empresarial; esto es, positiva en vez de negativamente, pero sin ser significativa en términos estadísticos para el caso de las personas jurídicas más ricas, especialmente las superricas y súper superricas.

Por estas razones no se detallan nuevamente los hallazgos ya mencionados a propósito de las Notas de investigación 14, 15 y 16.

La Nota de investigación 20 se concentra en realizar un análisis comparativo de la estructura tributaria de Colombia con la de otros países de referencia, con el fin de esclarecer el espacio fiscal potencial que existe para que el país pueda lograr una estructura y un esfuerzo tributarios deseables a la luz de su condición de economía de nivel de ingreso medio. Para ello se toma una misma fuente, confiable y consistente de información estadística, como la de la OCDE, mediante la cual se comparan las estructuras tributarias de Colombia con las de tres referentes bien diferenciados: Chile - país de ingreso medio y emblemático del modelo neoliberal aplicado desde los ochenta en América Latina-, Estados Unidos - país altamente desarrollado y con un Estado liberal tradicional- y el promedio de países de la OCDE, países en su mayoría desarrollados y con Estados de bienestar con diversos grados de profundidad.

El principal hallazgo consiste en que ante la muy elevada concentración del ingreso y la riqueza en cabeza de las personas jurídicas y naturales más ricas del país, y dado el espacio existente entre una tarifa efectiva potencial de referencia y la tarifa efectiva observada para estos grupos de personas declarantes, se deberán de incrementar progresivamente las tarifas efectivas del impuesto a la renta y de otros, como los del patrimonio y de dividendos, primordialmente para aquellos grupos de personas más ricas del país que hasta ahora han resultado beneficiadas del estatuto tributario, especialmente las superricas y super superricas -incluso con una rebaja sustancial de tarifas nominales, como condición de la eliminación de tratos preferenciales injustificados social y económicamente; y ello sin poner en riesgo la capacidad de inversión ni la competitividad de las empresas en Colombia-; además de que se debe poder contemplar la reducción progresiva de tarifas efectivas para personas y naturales con ingresos medios y bajos, para ir avanzando, así, hacia el objetivo de lograr progresividad, equidad y eficiencia adecuadas, así como de asegurar una razonable presión tributaria total - como la proporción del valor total de impuestos en el PIB-, consecuentes con la búsqueda de una deseable redistribución de la riqueza y el ingreso en el país y de una debida capacidad de las finanzas públicas, para afrontar aquellos retos inaplazables de política social, territorial y económica en una perspectiva perdurable.

En la Nota de investigación 21 se plantean algunos lineamientos básicos de referencia para la implantación de un proceso de reforma estructural de la tributación en Colombia, bajo los propósitos de: (i) avanzar

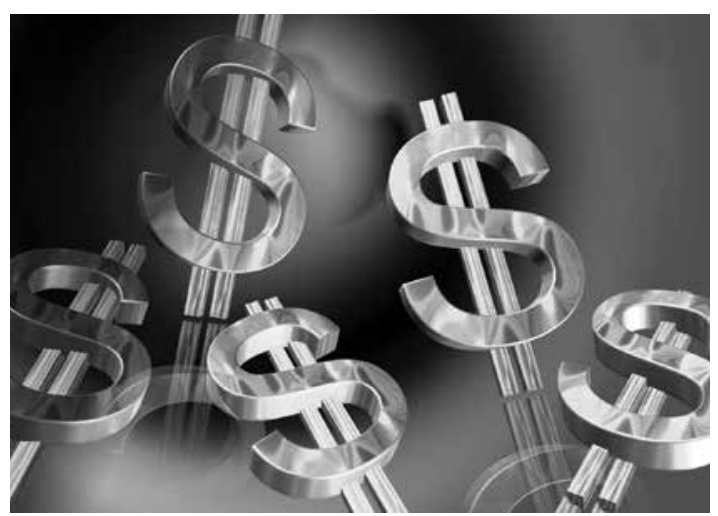


hacia el cumplimiento cabal de los principios constitucionales de equidad horizontal, progresividad vertical, eficiencia y transparencia administrativa; (ii) desmontar el sesgo prorrico, especialmente pro superrico y pro super superrico, prevaleciente en el estatuto tributario, mediante la eliminación de gran parte de los tratos preferenciales discriminatorios no justificados ni económica ni socialmente; con la consecuente elevación de las tarifas efectivas de tributación para las personas naturales y jurídicas más ricas, especialmente superricas y super superricas -incluso con una rebaja sustancial de tarifas nominales en muchos casos, y sin que se pueda desalentar la inversión y la competitividad productivas, ni deteriorar condiciones de vida digna-; (iii) reducir ostensiblemente la heterogeneidad y dispersión de las tarifas efectivas de tributación tanto inter e intrasectorialmente como inter e intraniveles de ingreso - según deciles, subdeciles y subdeciles de subdeciles, por ejemplo-; y, entre otros, (iv) robustecer las finanzas públicas para poder afrontar con cierta solvencia las prioridades sociales y económicas más acuciantes en el país, mientras que se asegura tanto una razonable estabilidad fiscal y macroeconómica - en una perspectiva perdurable-, como los niveles adecuados de crecimiento económico.

Algunas de las medidas propuestas como componentes básicos de la reforma estructural de la tributación en Colombia se detallan en la misma Nota de investigación 20, por lo que no vale la pena repetirlas en esta introducción.

Antes de terminar resulta indispensable recabar en la necesidad impostergable de imponer todos los correctivos que sean idóneos y eficaces para combatir la evasión y la elusión tributarias, tan elevadas y onerosas económica y socialmente para el país - aparte de las tres primeras mencionadas en el párrafo anterior-, concentrándose especial, aunque no exclusivamente, en aquellas personas naturales y jurídicas ricas que utilizan diversas figuras y mecanismos para evadir o eludir, como, por ejemplo, la

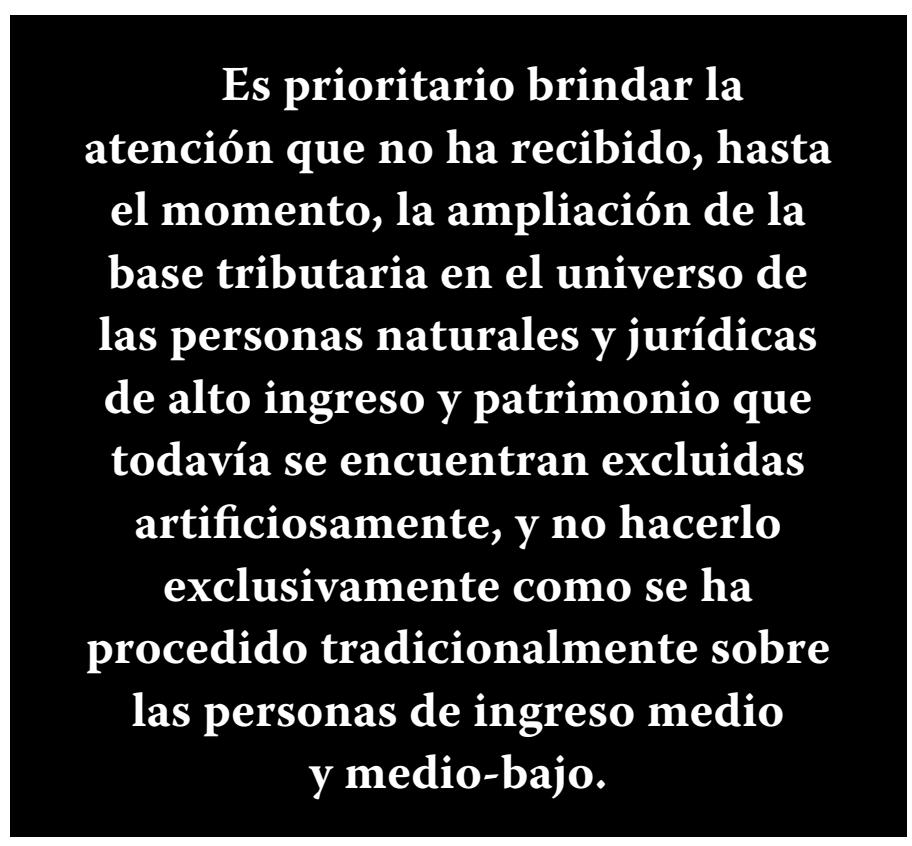

creación de "sociedades refugio", tanto en el exterior como en el país ${ }^{3}$. Es prioritario brindar la atención que no ha recibido, hasta el momento, la ampliación de la base tributaria en el universo de las personas naturales y jurídicas de alto ingreso y patrimonio que todavía se encuentran excluidas artificiosamente, y no hacerlo exclusivamente como se ha procedido tradicionalmente sobre las personas de ingreso medio y medio-bajo. En otras palabras, el esfuerzo tributario adicional que debe lograrse en el país no debería seguir sustentándose exclusivamente en las mismas personas declarantes ricas, sino, progresivamente y en especial, en aquellas personas muy pudientes que todavía permanecen excluidas de la base de tributación por

3 En este punto resulta ilustrativo, y por lo demás inquietante, mencionar que cerca de un $22 \%$ de las personas jurídicas declarantes ante la Dian en 2017 —es decir, 110000 de 500000 personas jurídicas declarantes, con código de actividad económica- declararon un ingreso total neto de cero, sin que muchas de ellas registraran aportes ni a la seguridad social ni a las entidades. 
el aprovechamiento de procedimientos irregulares (o abiertamente ilícitos y claramente ilegítimos).

Sin duda alguna, los principales hallazgos evidenciados en este segundo volumen brindan todavía más elementos de juicio para ratificar la necesidad pregonada en el primer volumen de avanzar en el compromiso y la implementación de un Pacto societal para la reforma estructural de la tributación y la fiscalidad en Colombia bajo principios rectores constitucionales y de economía política y política pública de inclusión social, como los recién referidos.

Para concluir esta introducción resulta indispensable hacer dos precisiones. La primera se refiere al hecho de que el análisis de desigualdad, concentración y distribución de ingresos y patrimonio se concentra exclusivamente en las personas naturales y jurídicas que declaran ante la Dian, dado que la fuente básica de la investigación son las declaraciones de renta presentadas ante esa autoridad tributaria. Por ello, es de esperar que los niveles de inequidad y de concentración, considerando la totalidad de la población del país, sean todavía mayores que los aquí consignados.

La segunda se basa en que la publicación del presente libro se ha efectuado justo en las primeras semanas de la irrupción de la pandemia del Covid-19 en Colombia, por lo que no se hace referencia a sus impactos sobre la agudización de la desigualdad, la pobreza y el desempleo, entre otros, ni al deterioro particular de las finanzas públicas en el país en una perspectiva perdurable. Esta última temática es, al menos en parte, abordada en un libro de los mismos autores y que se encuentra en proceso publicación, intitulado Medidas sociales y económicas de emergencia ante la pandemia del Covid-19 en Colombia. Visión desde una economía política de inclusión social. 